\title{
Detection of an extraordinarily large magnetic field in the unique ultra-cool Ap star HD 154708 ${ }^{\star}$
}

\author{
S. Hubrig ${ }^{1}$, N. Nesvacil ${ }^{1,2}$, M. Schöller ${ }^{1}$, P. North ${ }^{3}$, G. Mathys ${ }^{1}$, D. W. Kurtz ${ }^{4}$, \\ B. Wolff ${ }^{5}$, T. Szeifert ${ }^{1}$, M. S. Cunha ${ }^{6}$, and V. G. Elkin ${ }^{4}$
}

\author{
1 European Southern Observatory, Casilla 19001, Santiago 19, Chile \\ e-mail: shubrig@eso.org \\ 2 Department of Astronomy, University of Vienna, Türkenschanzstrasse 17, 1180 Vienna, Austria \\ ${ }^{3}$ Laboratoire d'Astrophysique, École Polytechnique Fédérale de Lausanne (EPFL), Observatoire, 1290 Sauverny, Switzerland \\ ${ }^{4}$ Centre for Astrophysics, University of Central Lancashire, Preston PR1 2HE, UK \\ 5 European Southern Observatory, Karl-Schwarzschild-Str. 2, 85748 Garching, Germany \\ ${ }^{6}$ Centro de Astrofísica da Universidade do Porto, Rua das Estrelas, 4150 Porto, Portugal
}

Received 7 June 2005 / Accepted 29 July 2005

\begin{abstract}
We have discovered an extraordinarily large mean longitudinal magnetic field of $7.5 \mathrm{kG}$ in the ultra-cool low mass Ap star HD 154708 using FORS 1 in spectropolarimetric mode. From UVES spectra, we have measured a mean magnetic field modulus of $24.5 \mathrm{kG}$. This is the second-largest mean magnetic field modulus ever measured in an Ap star. Furthermore, it is very likely that this star is one of the coolest and least massive among the Ap stars and is located in the H-R diagram in the same region in which rapidly oscillating Ap stars have been detected. We note that all known roAp stars have much smaller magnetic fields, by at least a factor of three.
\end{abstract}

Key words. stars: chemically peculiar - stars: polarization - stars: magnetic fields - stars: abundances stars: individual: HD 154708

\section{Introduction}

A large variety of physical processes occur in the atmospheres of upper main sequence stars that have not yet been fully incorporated into stellar models, or even securely identified and understood. These processes include convection, turbulence, meridional circulation currents, diffusion of trace elements within the dominant hydrogen plasma, and mass loss through stellar winds. The chemically peculiar stars, Ap and Bp stars, play a key role in our efforts to understand the relevant physics, since it is in these stars that the effects of the various processes acting below, in and above the stellar atmosphere are most clearly visible. Ap and Bp stars are main-sequence A and $\mathrm{B}$ stars in the spectra of which the lines of some elements are abnormally strong (e.g., $\mathrm{Si}, \mathrm{Sr}$, rare earths) or weak (in particular, He). Among Ap stars, the magnetic chemically peculiar stars are particularly important. For a long time, Ap stars were the only non-degenerate stars beside the sun in which direct detections of magnetic fields had been achieved.

Recently, we carried out a systematic search for magnetic fields in chemically peculiar stars whose magnetic field has

* Based on observations obtained at the European Southern Observatory, Paranal, Chile (ESO programmes Nos. 073.D-0464, 075.D-0145). never been studied before. The goal of this study was to statistically enlarge our data sample by including all southern stars for which the position in the H-R diagram is known from accurate Hipparcos (ESA 1997) parallaxes $(\sigma(\pi) / \pi<0.2)$ and from photometric data in the Geneva and Strömgren systems, used to determine their effective temperatures. In the course of this study we discovered an extreme magnetic Ap star, HD 154708 ( $=\mathrm{CD}-57^{\circ} 6753$ ), which has one of the strongest magnetic longitudinal fields ever detected in an Ap star. In the following we give details on our magnetic field measurements, the determination of the fundamental parameters of this star, and briefly discuss the appearance of the spectrum.

\section{Observations and magnetic field measurements}

Magnetic stars generally have large-scale organized magnetic fields that can be diagnosed through observations of circular polarization in spectral lines. Observations of the wavelength shift between spectral lines recorded in right-hand circular polarized light (RCP) and left-hand circular polarized light (LCP) with FORS 1 in spectropolarimetric mode have been the main source of stellar magnetic field measurements during recent years at the VLT (e.g., Bagnulo et al. 2002; Hubrig et al. 2004). Most Ap and Bp stars have magnetic fields which, in first approximation, are dipolar; the dipole axis is generally 


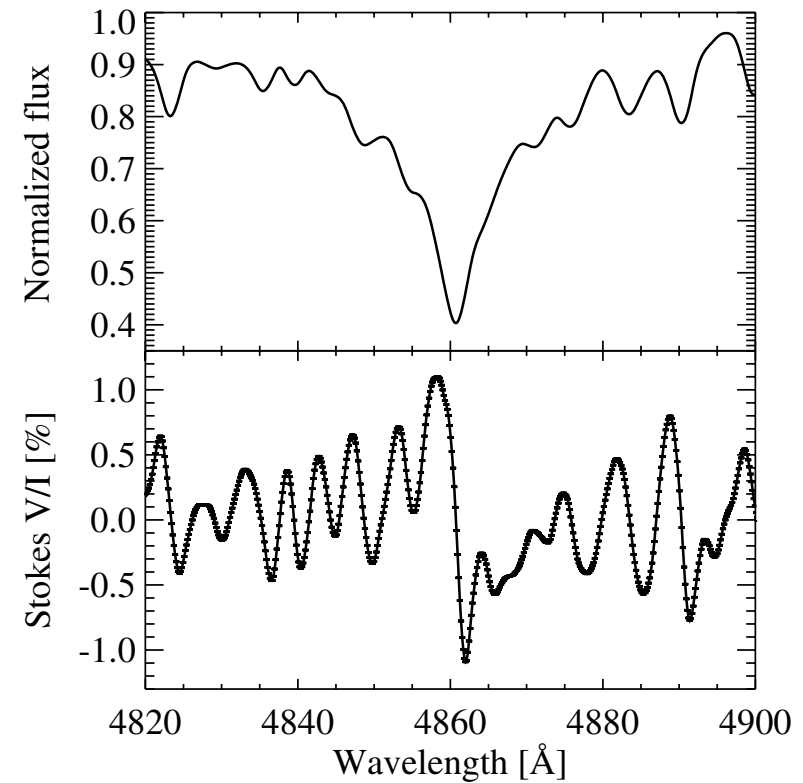

Fig. 1. Stokes $I$ and $V / I$ spectra of the Ap star HD 154708 in the vicinity of the $\mathrm{H} \beta$ line.

inclined with respect to the stellar rotation axis. This simple dipolar structure is very favourable for observations of a global wavelength shift of disk-integrated spectral lines between RCP and LCP.

We have obtained a longitudinal field determination for HD 154708 with FORS 1 at the VLT, which is a multi-mode instrument equipped with polarization analyzing optics comprising super-achromatic half-wave and quarter-wave phase retarder plates, and a Wollaston prism with a beam divergence of $22^{\prime \prime}$ in standard resolution mode. We used a non-standard readout mode with low gain (A, $1 \times 1$, low) and the GRISM 600B with the narrowest available slit width of 0.4 in the wavelength range $3480-5890 \AA$ to cover all hydrogen Balmer lines from $\mathrm{H} \beta$ to the Balmer jump at a spectral resolution of $R \sim$ 2000. The determination of the mean longitudinal fields using FORS 1 is described in detail in Bagnulo et al. (2002) and Hubrig et al. (2004). Eight spectra of HD 154708 in circular polarized light using two different retarder waveplate setups $\left(\alpha=+45^{\circ}\right.$ and $\left.-45^{\circ}\right)$ at $S / N>1000$ have been recorded on 2004 September 19. The measurements of the circular polarization of opposite sign induced in the wings of each Balmer line by the Zeeman effect revealed an extraordinarily large longitudinal magnetic field $\left\langle B_{z}\right\rangle=7.5 \pm 0.05 \mathrm{kG}$. Figure 1 shows both, the Stokes $I$ and $V / I$ spectra of this star in the vicinity of the $\mathrm{H} \beta$ line.

HD 154708 has never been studied in detail in the past and there are only four references in the SIMBAD database. It is very likely that it is one of the coolest and least massive Ap stars known. Using Geneva photometry (Rufener 1988; Burki et al. 2005) and the Hipparcos parallax, we obtain for HD $154708 T_{\text {eff }}=6800 \mathrm{~K}$ and $\log g=4.11 \pm 0.15$ (assuming a $\pm 300 \mathrm{~K}$ uncertainty on $T_{\text {eff }}$ ), using the $T_{\text {eff }}$ calibration of the $B 2-G$ index (Hauck \& North 1993, Eq. (2)) which has to be slightly extrapolated here: according to this colour index, this star is clearly among the coolest Ap stars. The $u v b y \beta$ colours
(Knude 1977) lead to a hotter temperature $T_{\text {eff }}=7500 \mathrm{~K}$ through the calibration of Napiwotzki et al. (1993), but they are severely distorted by the peculiar nature of the star, especially through the $\lambda 5200$ depression. The stellar mass of $1.5 \pm 0.1 M_{\odot}$ results from interpolation of the evolutionary tracks of Schaller et al. (1992) for a solar metallicity $Z=0.020$ as explained by North et al. (1997), though without the Lutz-Kelker correction, which is smaller than the error bar. This star with a mass of $1.5 M_{\odot}$ lies far beyond the cooler edge of the empirically determined $\delta$ Scuti instability strip and shares the region in the H-R diagram with two of the coolest and lowest mass rapidly oscillating Ap stars, HD 101065 and HD 217522, pulsating with periods of $12.1 \mathrm{~min}$ and $11.5 \mathrm{~min}$, respectively (Hubrig et al. 2000a; Kurtz 1978; Kurtz 1983).

The rapidly oscillating Ap stars (roAp) were the first stars for which high-overtone $\mathrm{p}$-mode pulsations have been definitely detected and are, therefore, prime candidates for asteroseismological studies. They are found among the cool magnetic chemically peculiar stars and pulsate with periods between 5.6 and 21 min with photometric $B$ amplitudes of tenths of a mmag up to $6 \mathrm{mmag}$, and radial velocity amplitudes of a few $\mathrm{m} \mathrm{s}^{-1}$ up to $5 \mathrm{~km} \mathrm{~s}^{-1}$. The indirect effect of the magnetic field on pulsations, through the suppression of envelope convection, seems to be of prime importance to the excitation of the oscillations observed in roAp stars (Balmforth et al. 2001; Cunha 2002). Moreover, the direct effect of the magnetic field also leads to a selective damping that may be responsible for the fact that only certain modes are excited in certain stars (Cunha \& Gough 2000; Saio 2005). There are 34 such stars currently known. Detailed reviews of the photometric results for roAp stars have been published by Kurtz (1990), Matthews (1991), and Kurtz \& Martinez (2000). The radial velocity studies have not yet been reviewed; see Mkrtichian \& Hatzes (2005), Kurtz et al. (2005), Kochukhov (2004), Kochukhov \& Ryabchikova (2001) for some examples of the rich results coming from the radial velocity studies. It is clear that high resolution spectroscopy is more powerful and sensitive than photometry to detect and characterise pulsations in roAp stars.

Figure 2 shows the distribution of roAp and non-oscillating Ap (noAp) stars with available Hipparcos parallaxes in the astrometric H-R diagram (Arenou \& Luri 1999), taken from Hubrig et al. (2000a). The $a_{V}$ values of the Ap stars can be compared to those of the theoretical evolutionary tracks, since the bolometric correction of cool Ap stars differs little from the standard one. HD 154708 is indicated by a 6-branch star at two positions: one (to the left) corresponding to the $u v b y \beta$ effective temperature of $7500 \mathrm{~K}$, the other (to the right) corresponding to the Geneva $B 2-G$ temperature of $6800 \mathrm{~K}$, assuming $E(B-V) \sim 0.05$ or $E(B 2-G) \sim 0.06$, suggested by the reddening maps of Lucke (1980).

A large number of spectra for a search of oscillations in HD 154708 have been taken with UVES at the VLT on 2005 May 19 , in a short spectral region (5000-7000 $)$ at a resolving power of $\lambda / \Delta \lambda \approx 1.1 \times 10^{5}$. We used the average spectrum at $S / N \sim 140$ which is the average of all time series spectra taken with short exposures for the measurement of the mean magnetic field modulus and for a preliminary abundance analysis. As expected, most spectral lines appear resolved into 


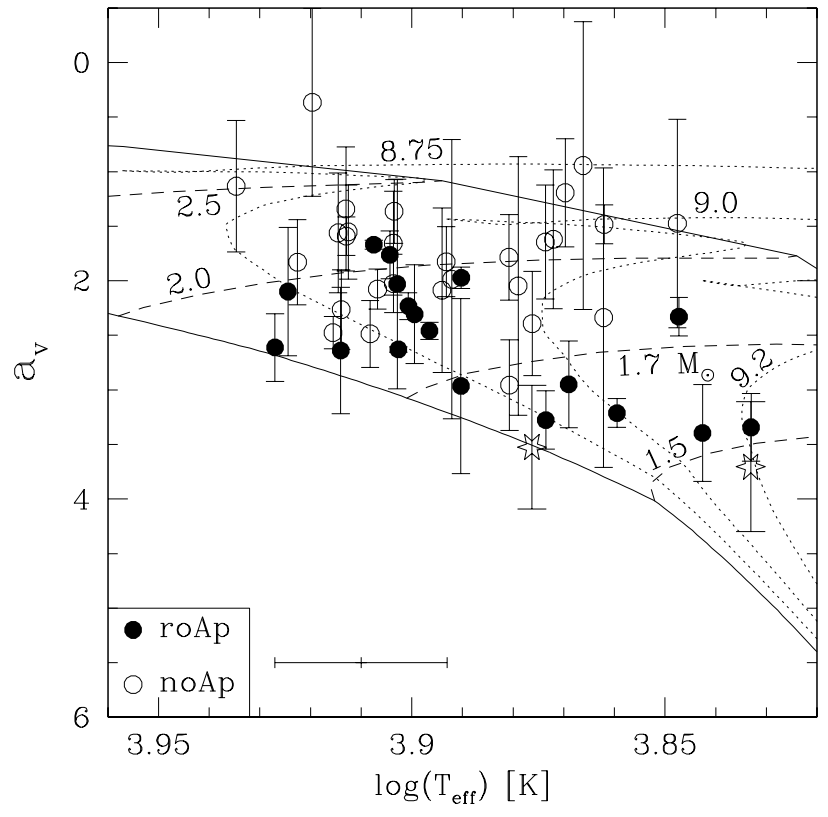

Fig. 2. Astrometric HR-diagram of the roAp (full dots) and of the noAp stars (open dots), where $a_{V}=\pi \cdot 10^{0.2\left(m_{V}-A_{V}+5\right)}$ (see Arenou $\&$ Luri 1999). The lower continuous curve (ZAMS) is an isochrone at $\log t=5.7$ based on the models of Schaller et al. (1992) for $Z=0.020$. The dotted curves are the isochrones at the indicated $\log t$, while the dashed curves are the main sequence parts of the evolutionary tracks for masses between 1.5 and $2.5 M_{\odot}$. HD 154708 is indicated by a 6-branch star at two positions: one (to the left) corresponding to the uvby $\beta$ effective temperature of $7500 \mathrm{~K}$, the other (to the right) corresponding to the Geneva B2-G temperature of $6800 \mathrm{~K}$.

magnetically split components (Fig. 3), so that we can easily measure the mean magnetic field modulus from the separation of the $\sigma$, or $\sigma$ and $\pi$ components. Taking an average of all measurements we obtain $\langle B\rangle=24.5 \pm 1.0 \mathrm{kG}$. We should note that this is the second highest value of a field modulus ever measured in Ap and Bp stars. Up to now, the number of magnetic stars with a measured mean magnetic field modulus using magnetically resolved lines was 47 . But only one star in this sample, HD 215441 of BpSi spectral type, has a surface field larger than $20 \mathrm{kG}$ (Babcock 1960).

The magnetic field of HD 154708 is so strong that we are not able to interpret the spectrum in the region where the Fe II lines $\lambda 6147.7$ and $\lambda 6149.2$, which belong to the same multiplet, are generally observed in Ap stars. Features corresponding to the transitions from which these lines arise are probably present in the spectrum of HD 154708, but distorted beyond recognition by partial Paschen-Back effect (Fig. 3). Accordingly, the Zeeman doublet Fe II $\lambda$ 6149.2, which is one the most often used diagnostics of the mean magnetic field modulus in Ap and Bp stars (Mathys et al. 1997), cannot be exploited here for determination of this field moment, due to the very strong magnetic field. Instead it should be modeled accounting properly for the physics of the partial Paschen-Back effect. This will be the subject of future work. Our estimate of the mean magnetic field modulus was based on the measurement of the Nd III $\lambda 6145.1$ line (see Fig. 3) with an effective Landé factor $g_{\mathrm{eff}}=1.125$ and a few other suitable lines

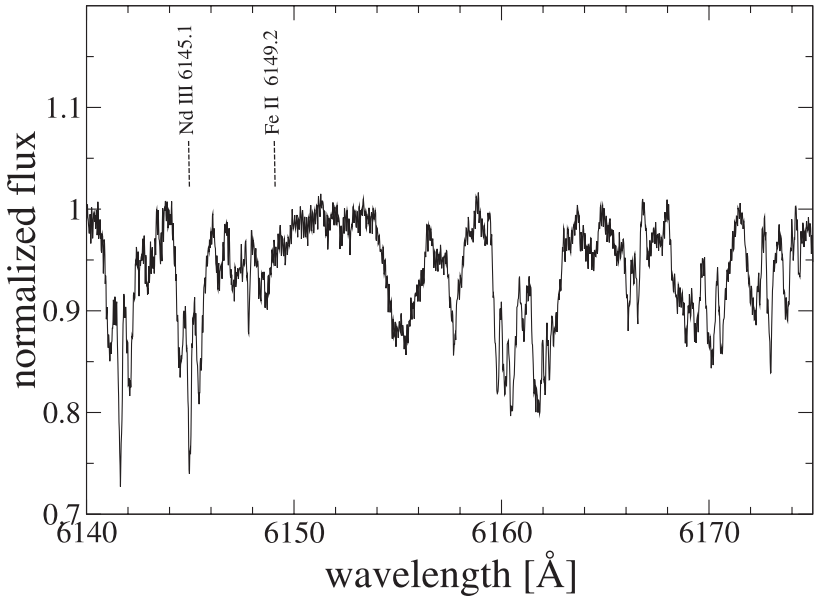

Fig. 3. The UVES spectrum of HD 154708 in the spectral region around the Fe II lines $\lambda 6147.7$ and $\lambda 6149.2$, which are distorted beyond recognition by partial Paschen-Back effect, and the line Nd III $\lambda 6145.1$, which is used to estimate the mean magnetic field modulus.

of iron-peak elements with simple Zeeman patterns, using a Gaussian and a centre-of-gravity method.

The $v \sin i$ value of this star is very low. The best fit to the observed spectral line profile of the magnetically insensitive line Fe I $\lambda 5434.5$ results in $v \sin i=1.0 \mathrm{~km} \mathrm{~s}^{-1}$. Our preliminary abundance analysis in the available short spectral region indicates that abundances of iron peak elements and rare-earth elements are very similar to those of roAp stars. The study of the rare-earth elements $\mathrm{Pr}$ and $\mathrm{Nd}$ reveals a difference of about 1 dex in the abundances derived from the first and second ions. This Pr-Nd anomaly is usually explained by vertical abundance stratification of these elements in the stellar atmosphere and is a strong indicator that the star is expected to be a roAp star (e.g., Ryabchikova et al. 2004).

\section{Discussion and outlook}

Strongly magnetic stars are of special interest because only in these stars the effect of the magnetic field on the stellar atmospheres can be studied with the greatest detail and accuracy. The information on the very strong magnetic field, together with the results on vertical and horizontal abundance inhomogeneities of various chemical elements should be used to calculate a realistic model atmosphere for HD 154708 which will be confronted with the predictions of current diffusion models. The presence of such a huge magnetic field in a very cool Ap star is especially intriguing in view of the previous studies of the strength of magnetic fields in Ap and Bp stars of different mass and effective temperature (Thompson et al. 1987; Mathys et al. 1997; Hubrig et al. 2000b) which showed that the hotter and more massive Bp stars tend to exhibit stronger magnetic fields.

An additional interest to study this star arises from its position in the H-R diagram (Fig. 2). Because of the location of HD 154708 in the same region of parameter space in which rapidly oscillating Ap stars have been detected, this star is an excellent candidate for a search of variations of radial velocities 
and field modulus through a possible pulsation cycle and to study radial distribution of the pulsational amplitudes and phases through the atmosphere. HD 154708 was searched on five separate occasions photometrically by Martinez \& Kurtz (1994) and also by Handler \& Paunzen (1999) with no detected oscillations in any case. But, as was pointed out in the previous section, HD 154708 shows the ionisation disequilibrium typical of roAp stars and high precision radial velocity studies are much more sensitive for detecting low amplitude pulsation in roAp stars. A study is underway of the radial velocities using the 121 spectra (obtained with a time resolution of $63 \mathrm{~s}$ ) that were averaged to produce Fig. 3. Its results will be reported in a future publication. If pulsations are detected, it will be the first roAp star with such an extremely large mean longitudinal magnetic field and mean field modulus.

Certainly, additional observations of this extreme magnetic Ap star over a much larger spectral region should be conducted to determine the magnetic field geometry and to carry out a careful multi-element abundance analysis. Such an analysis is also important for a comparison with the abundance pattern and the behaviour of the magnetic field in other cool pulsating Ap stars. The confirmation of an inconsistency between abundances derived from weak and strong spectral lines and the lines of different ions would provide clear evidence for prominent vertical abundance stratification of certain chemical elements which is frequently found in roAp stars. Also the possible presence of radial gradients of the magnetic field in the atmosphere of HD 154708 should be critically examined by measurements of the mean magnetic field modulus from spectral lines resolved into magnetically split components lying on different sides of the Balmer jump, in a similar way as has been done by Nesvacil et al. (2004). We have recently applied for VLT director's discretionary time to obtain UVES spectra over the range 3030-9460 ^ with the highest spectral resolution and at high $S / N$, but unfortunately the DDT committee found this science not interesting.

Acknowledgements. M.S.C. is supported by FCT and FEDER (through POCI2010) through the project POCTI/CTE-AST/ 57610/2004. This research has made use of the SIMBAD database, operated at CDS, Strasbourg, France.

\section{References}

Arenou, F., \& Luri, X. 1999, ASP Conf. Ser., 167, 13

Babcock, H. W. 1960, ApJ, 132, 521

Bagnulo, S., Szeifert, T., Wade, G. A., et al. 2002, A\&A, 389, 191

Balmforth, N. J., Cunha, M. S., Dolez, N., et al. 2001, MNRAS, 323, 362

Burki, G. et al. 2005, GENEVA photometric database http://obswww. unige.ch/gcpd/ph13.html

Cunha, M. S., \& Gough, D. O. 2000, MNRAS, 319, 1020

Cunha, M. S. 2002, MNRAS, 333, 47

ESA 1997, The Hipparcos and Tycho Catalogues, ESA SP-1200, Noordwijk

Handler, G., \& Paunzen, E. 1999, A\&AS, 135, 57

Hauck, B., \& North, P. 1993, A\&A, 269, 403

Hubrig, S., Kharchenko, N., Mathys, G., \& North, P. 2000a, A\&A, 355,1031

Hubrig, S., North, P., \& Mathys, G. 2000b, ApJ, 539, 352

Hubrig, S., Szeifert, T., Schöller, M., et al. 2004, A\&A, 415, 685

Knude, J. K. 1977, A\&AS, 30, 297

Kochukhov, O., \& Ryabchikova, T. 2001, A\&A, 374, 615

Kochukhov, O. 2004, ApJ, 615, L149

Kurtz, D. W. 1978, IBVS, 1436

Kurtz, D. W. 1983, MNRAS, 205, 3

Kurtz, D. W. 1990, ARA\&A, 28, 607

Kurtz, D. W., \& Martinez, P. 2000, Baltic Astron., 9, 253

Kurtz, D. W., Elkin, V. G., \& Mathys, G. 2005, MNRAS, 358, L6

Lucke, P. B. 1990, A\&A, 90, 350

Martinez, P., \& Kurtz, D. W. 2004, MNRAS, 271, 129

Mathys, G., Hubrig, S., Landstreet, J. D., et al. 1997, A\&AS, 123, 353

Matthews, J. M. 1991, PASP, 103, 5

Mkrtichian, D. E., \& Hatzes, A. P. 2005, A\&A, 430, 263

Napiwotzki, R., Schönberner, D., \& Wenske, V. 1993, A\&A, 268, 653

Nesvacil, N., Hubrig, S., \& Jehin, E. 2004, A\&A, 421, L51

North, P., Jaschek, C., Hauck, B., et al. 1997, Hipparcos - Venice '97, ESA SP-402, 239

Rufener, F. 1988, GENEVA Photometric Catalogue, 4th edition, Observatoire de Genève

Ryabchikova, T., Nesvacil, N., Weiss, W. W., et al. 2004, A\&A, 423, 705

Saio, H. 2005, MNRAS, 360, 1022

Schaller, G., Schaerer, D., Meynet, G., \& Maeder, A. 1992, A\&AS, 96, 269

Thompson, I. B., Brown, D. N., \& Landstreet, J. D. 1987, ApJS, 64, 219 